\title{
MK801 Regulates the Expression of Key Osteoarthritis Factors in Osteoarthritis Synovial Fibroblasts Through Complement C5
}

\section{Zhi Huang}

Guizhou Medical University

\section{Yuanyu Feng}

Guizhou Medical University

Xiaoxi Zhu

Guizhou Medical University

Lin Wang

Guizhou Medical University

Wei Lu ( $\square$ adjqy@126.com )

Guizhou Medical University

Research article

Keywords: OA, C5, MK801, NF-KB, inflammatory factors

Posted Date: December 29th, 2020

DOI: https://doi.org/10.21203/rs.3.rs-135712/v1

License: (c) (1) This work is licensed under a Creative Commons Attribution 4.0 International License. Read Full License 

osteoarthritis synovial fibroblasts through complement C5

3 Zhi Huang ${ }^{1 *}$, Yuanyu Feng ${ }^{2 *}$, Xiaoxi Zhu ${ }^{3}$, Lin Wang ${ }^{4 \triangle}$, Wei $\mathrm{Lu}^{2,5 \#}$

$4{ }^{1}$ Department of interventional radiology, the Affiliated Cancer Hospital of Guizhou Medical

5 University, Guiyang 550002, P.R. China

$6 \quad{ }^{2}$ Department of Anesthesiology, Guizhou Medical University, Guiyang 550002, P.R. China

$7 \quad{ }^{3}$ School of Basic Medical Sciences, Guizhou Medical University, Guiyang 550002, P.R. China

$8 \quad{ }^{4}$ Department of pain, Affiliated Hospital of Guizhou Medical University, Guiyang 550002, P.R.

9 China

$10 \quad{ }^{5}$ Lead Contact

$11{ }^{\triangle}$ Co-corresponding author

$12 *$ These authors contributed equally to this work

13 \#Corresponding authors:

14 Wei Lu

15 Department of Anesthesiology, Guizhou Medical University, Guiyang city Beijing Road 9\# 16 550002, P.R. China

17 E-mail: adjay@126.com

18 Phone: 86-0851-86873981

19 Fax: 86-0851-86873981 


\section{Abstract}

Background: Osteoarthritis is currently one of the most common chronic diseases. As life expectancy increases, its prevalence and incidence are expected to rise. At present, more and more evidences prove the correlation between the complement system and OA. This study aims to investigate complement C5's influence on the effect of MK801 on osteoarthritis synovial fibroblasts (OA-SFs).

Methods: We used IL-1b to induce OA-SFs derived from mice to obtain OA-SFs. And we performed RT-PCR and Western Blot assays to evaluate the expression levels of associated mRNA and protein. The alteration of MAC expression on OA-SFs cell membrane was evaluated by immunofluorescence assay. The expression of related inflammatory factors of OA-SFs was evaluated by ELISA experiment.

Results: MK801 could significantly inhibit the expression of osteoarthritis (OA) marker factors, such as: membrane attack complex (MAC), tumor necrosis factor- $\alpha$ (TNF- $\alpha)$ and matrix metalloproteinase-13 (MMP13). Meanwhile, MK801 can significantly inhibit the expression of complement C5 (C5) in OA-SFs. Immunofluorescence assay showed that MAC expression on OA-SFs cell membrane was significantly inhibited by MK801. The nucleo-plasmic separation experiment demonstrated that MK801 could significantly inhibit the activation of Nuclear factor- $\kappa \mathrm{B}(\mathrm{NF}-\kappa \mathrm{B})$ signaling pathway in OA-SFs. Futhermore, koncking down the the expression of C5 reversed the inhibition MK801 on the expression of OA-SFs inflammatory factors.

Conclusions: These results illustrated two points: first, MK801 inhibited the generation of MAC and the release of inflammation factors in OA-SFs through C5; second: MK801 inhibited the activation of NF- $\kappa \mathrm{B}$ signaling pathway in OA-SFs.

Key words: OA, C5, MK801, NF-кB, inflammatory factors 


\section{Background}

Osteoarthritis (OA), as the most common arthritis, is one of the main causes of disability worldwide[1]. Currently, OA is considered to be a kind of cartilage matrix destruction, which is caused by a complex interaction of a series of biochemical and biomechanical factors that occur simultaneously[2]. Although the research on OA mainly focuses on the pathogenesis of articular cartilage destruction, the biological and morphological changes caused by it are not limited to articular cartilage. Synovium, subpatellar fat, ligaments and bone are also affected[3-7]. In addition, there is increasing evidence that although cartilage degradation is the earliest event, synovial inflammation involves many signs and symptoms of OA, including joint swelling and fluid accumulation[8, 9].

Joint swelling due to excessive joint burden leads to cartilage inflammation with subsequent degradation mediated by the release of inflammation and ECM degradation factors such as IL-1 $\beta$, TNF- $\alpha$ and MMP-1, MMP-3, and MMP-9[10, 11]. Current studies have shown that in addition to overload and joint instability, various factors released from joint-related tissues, such as catabolism, inflammation, or damage to tissue integrity, are key factors for OA[12, 13]. Further more, excessive production of inflammatory synovial cytokines and growth factors may play a crucial role in the pathophysiology of OA. The secretion of pro-inflammatory cytokines, mainly IL- $1 \beta / \mathrm{TNF}-\alpha$, is one of the key steps in the regulation of abnormal degenerative processes common in the pathophysiology of OA, in which fibroblast-like synovial cells (FLS) play a key role[14-17]. The important role of IL-1 $\beta$ and TNF- $\alpha$, which are both pro-inflammatory and metabolically decomposing cytokines, in the pathogenesis of OA, has been widely accepted in the past decades[18-20].

At present, more and more evidences prove the correlation between the complement system and OA, such as complement stimulation affects the expression of inflammation and degradation molecules in OA chondrocytes, and the deposition of cell membrane attack complex (MAC) on the surface of chondrocytes[21-24]. In addition, Wang Q, etal. found that A significant increase in complement expression 
was found in synovial and membrane specimens of human OA patients, and the expression of inflammatory markers in mice lacking central complement component C5 was significantly reduced compared with wild-type mice in the OA model[21]. Despite these novel results, the signaling pathways of complement activation in the pathogenesis of OA remain unclear, so a better understanding of the interrelationships between the complement system and various other inflammatory and non-inflammatory factors in the OA scenario is urgently needed.

MK-801 (dizocilpine), an antagonist of the N-Methyl-D-aspartate (NMDA) receptor, has well known to inhibit activation NMDA signaling cascade[25, 26]. E.M. Kawamoto Wang Q, etal. found that MK-801 can inhibit the activition of NF- $\kappa \mathrm{B}$ signaling pathway in the Rat Hippocampus[27]. In addition, recent evidence suggests that MK-801 can block the release of a normal complement of neurons. Whilst some research has been carried out on the relationship between MK-801 and NF- $\mathrm{BB}$ signaling pathway, complement system, there is still very little scientific understanding of the role of this relationship in OA. Therefore, the specific objective of this study was to investigate the effect of MK801 on OA based on the complement system. And this paper attempts to show the possibilityof MK801 in OA therapy.

(1)




\section{Methods}

\section{Reagent}

DMEM medium (SH30022.01B), FBS (SH30087.01), penicillin (SH30010) and PBS (SH30256.01B) were brought from GETM Hyclone company. Antibodies: Anti-C5 (ab17930), Anti-GAPDH (ab8245), Anti-C5b-9 (ab55811), Anti-TNFA (ab92324), Anti-MMP13 (ab51072), Anti-Lamin B1 (ab16048), Anti-NF-kB-p65 (ab16502) and Anti-NFkB-p105/p50 (ab32360) were purchased from Abcam Co., Ltd, Cambridge, UK.

\section{Acquisition and culture of SFs}

The acquisition of SFs is operated as described in "protocol for the culture and isolation of murine synovial fibroblasts"[28]. In brief, the synovial tissues from total 6 10-week-old male C57BL/6 mice (Beijing Vital River Laboratory Animal Technology Co., Ltd.) were transferred to a $60 \mathrm{~mm}$ Petri dish containing $2 \mathrm{ml}$ DMEM. The synovium was then transferred to a $1.5 \mathrm{ml}$ Eppendorf tube containing $0.5 \mathrm{ml}$ DMEM and $0.5 \mathrm{ml} 1 \%$ type IV collagenase. After incubating for 1 hour at $37^{\circ} \mathrm{C}$ with constant temperature shaking $(200 \mathrm{rpm})$, vortex for 1 minute, and then resuspended with DMEM medium (SH30022.01B, Hyclone, USA) containing with 10\% FBS (SH30087.01, Hyclone, USA), $100 \mathrm{U} / \mathrm{ml}$ penicillin (SH30010, Hyclone, USA) and $100 \mathrm{mg} / \mathrm{ml}$ streptomycin. The cells were seeded into a $75-\mathrm{cm}^{2}$ flask and placed in a humidified atmosphere at $37^{\circ} \mathrm{C}$ with $5 \% \mathrm{CO} 2$. After the experiment was completed, the mice were sacrificed by carbon dioxide euthanasia.

\section{Induction of OA-SFs and vector transfection}

Cells obtained from the synovium were maintained in DMEM medium containing with $10 \% \mathrm{FBS}, 100 \mathrm{U} / \mathrm{ml}$ penicillin and $100 \mathrm{mg} / \mathrm{ml}$ streptomycin in a humidified atmosphere at $37^{\circ} \mathrm{C}$ with $5 \% \mathrm{CO}_{2}$. After subculturing the cells for 3-4 generations, add $10 \mathrm{~nm}$ IL-1 $\beta$ to stimulate the cells for $72 \mathrm{~h}$ to obtain OA-SFs. C5 shRNA or overexpression vector was transfected into OA-SFs using Lipofectamine 2000 (Cat.No.11668019, Invitrogen) according to the manufacturer's instruction. In brief, $1 \mu \mathrm{g}$ of C5 shRNA vector (CAT\#: TL511576, OriGene Technologies Inc) or $1 \mu \mathrm{g}$ of C5 overexpression vector was (CAT\#: MR225064, OriGene Technologies Inc) diluted 
with $100 \mu$ l Opti-MEM, as called fluid A. Fluid B was Opti-MEM contained 1ul of Lipofectamine 2000, and dissolved for 5 minutes before mixed with fluid A. Transfection reagent was added into OA-SFs were plated in 24-well plates which was transferred into Opti-MEM medium 24 hours before transfection. After 4 to 6 hours of reaction, culture medium was changed into DMEM full culture medium. Gene expression test was performed by qPCR 36 hours after transfection.

\section{Realtime PCR}

Total mRNA was isolated from OA-SFs using the Trizol reagent (\#15596018) (Life Technologies, USA) and then reversed transcribed by the QuantiTect Reverse Transcription Kit (\#205313) (Qiagen, Shanghai, China). Real-Time PCR was performed by the StepOnePlus system (Applied Biosystem, USA) using Thermo Fisher Scientific Maxima SYBR Green/ROX qPCR Master Mix assay (2X) (\#K0221). Primer sequences were showed in Table 1.

\section{Table1 Sequence of primers}

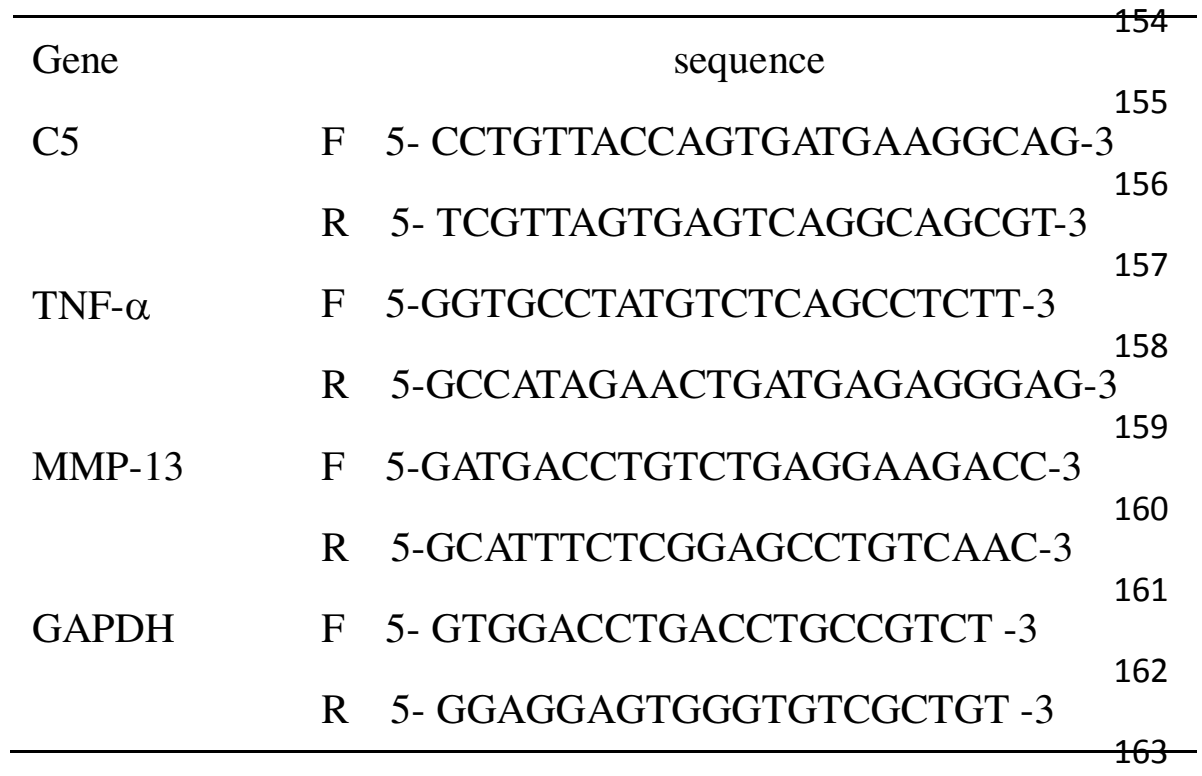

\section{Western Blot}

To detect cellular level of target proteins, protein extracted from OA-SFs were detected by Western Blot. Whole cell lysates were extracted by using the lysis buffer: $50 \mathrm{mM}$ Tris pH7.4, $150 \mathrm{mM} \mathrm{NaCl}, 1 \mathrm{mM}$ EDTA, 1\% Triton, and 10\% Glycerol along with protease and phosphatase inhibitor cocktail (Roche, Basel, Switzerland) protein concentrations were determined by the Bradford assay. Soluble proteins (30 40 $\mu \mathrm{g})$ 
were subjected to SDS-polyacrylamide gel electrophoresis. Separated proteins were electrophoretically transferred onto polyvinylidene difluoride (PVDF) membranes (Millipore, Billerica, MA,USA). Primary antibodies used in the present study were diluted into 5\% nonfat milk as1:500.

\section{Immunofluorescence(IF)}

Cell in each group was washed with PBS with $4 \mathrm{~g} / \mathrm{ml}$ concentration of DDP, and then treated with $4 \%$ paraformaldehyde at room temperature for $15 \mathrm{~min}$, washed with PBS buffer three times, added $0.2 \%$ Triton-X100 for permeabilization for $5 \mathrm{~min}$, washed with PBS buffer three times, sealed with PBS buffer containing 5\%BSA for $1 \mathrm{~h}$, washed with PBS buffer three times, and cell was subjected to rabbit monoclonal antibody C5b-9 (ab55811, 1:200, Abcam, Shanghai, China) incubated overnight at $4^{\circ} \mathrm{C}$, washed with PBS buffer solution for 3 times, incubated with Alexa Fluor 488-combined with the corresponding secondary antibody for 1 hour, washed with PBS buffer solution for 3 times, incubated with DAPI buffer solution(C1002 ,Beyotime Biotechnology,Shanghai China.) for 5min, and washed with PBS buffer solution for 3 times.Finally, the cells were observed and analyzed using the German Zeiss LSM710 confocal microscopy

\section{ELISA assay}

ELISA assay was performed using SimpleStep ELISA Kit of TNF-a (ab212073, Abcam) or IL-1 $\beta$ (ab197742, Abcam) according to the manufacturer's instruction. In brief, prepared all reagents, samples, and standards as instructed. Added $50 \mu \mathrm{L}$ standard or sample to appropriate wells, and then added $50 \mu \mathrm{L}$ Antibody Cocktail to all wells and incubated at room temperature for 1 hour. Aspirated and washed each well three times with $350 \mu \mathrm{L} 1 \mathrm{X}$ Wash Buffer. Add $100 \mu \mathrm{L}$ TMB Development Solution to each well and incubated for 10 minutes. Added $100 \mu \mathrm{L}$ Stop Solution and read OD at $450 \mathrm{~nm}$.

\section{Statistical Analysis}

All experiments in this study were repeated at least two times and average values of three experiments were presented as the mean standard seviation (SD) calculated by STDEV formula in Excel. The significance of all data was estimated by a Tukey's 
multiple-comparison test in the ANOVA analysis using the SigmaStat 3.5 software. Importantly, statistical significance was accepted when $P<0.05$.

\section{Results}

\section{MK801 inhibited the expression of OA marker factors in OA-SFs}

We performed western blot assay to analyze the impact of MK801 on OA marker factors protein expression levels in OA-SFs. We found that MK801 can significantly inhibit the protein expression levels of OA marker factors (MAC, TNF- $\alpha$, C5 and MMP13) in comparsion to the control group (Figure 1A\&B). And NMDA receptor agonist: NMDA (N-Methyl-D-aspartic acid) could significantly increase these protein expression levels in OA-SFs (Figure 1A\&B). Consistent with this, the mRNA expression levels of these factors were significantly inhibited by MK801, which was significantly increased by NMDA as well (Figure 1C\&D). Subsequently, we performed ELISA assay to detect the effect of MK801 on the release of inflammatory factors (TNF- $\alpha$ and IL-1 $\beta$ ) from OA-SFs. As shown in Figure 1D\&E, MK801 could significantly inhibit the release of TNF- $\alpha$ and IL-1 $\beta$, and NMDA could significantly increase the release of TNF- $\alpha$ and IL-1 $\beta$. These results indicated that MK801 could inhibit OA phenotype and inflammatory response in OA-SFs.

\section{MK801 inhibited the activation of NF- $\mathrm{KB}$ signaling pathway in OA-SFs}

As $\mathrm{NF}-\kappa \mathrm{B}$ signaling pathway is a known inflammatory response-related signaling pathway, and it has been reported that MK801 can inhibit the activation of NF- $\mathrm{BB}$ signaling pathway[29]. We speculated that MK801's inhibition of inflammatory response in $\mathrm{OA}-\mathrm{SFs}$ might be related to $\mathrm{NF}-\kappa \mathrm{B}$ signaling pathway. We performed nucleocytoplasmic separation experiments and found that compared with the control group, mk801 can significantly cause the accumulation of p65 and p50 in the cytoplasm, while significantly inhibiting p50 and p65 of entering the nucleus (Figure $2 \mathrm{~A} \& \mathrm{~B})$. These results indicated that MK801 inhibited the activation of NF- $\kappa \mathrm{B}$ signaling pathway in OA-SFs.

\section{MK801 inhibited MAC expression on OA-SFs cell membrane}

Due to the formation of MAC on the cell membrane, it is a symbolic event of OA[30].

In order to evaluate the effect of MK801 on the formation of MAC in OA-SFs cell 
membrane, we performed immunofluorescence assay to detect the expression of MAC on the cell membrane of OA-SFs. We found that MK801 can significantly inhibit the protein expression levels of OA marker factors (MAC, TNF- $\alpha$, C5 and MMP13) in comparsion to the control group, and this inhibition could be significantly reversed by NMDA (Figure $3 \mathrm{~A} \& \mathrm{~B}$ ). As shown in Figure 3C, the formation of MAC requires the participation of $\mathrm{C} 5 \mathrm{~b}[30]$. Therefore, we next tried to evaluate the role of C5 in MK801 inhibiting the phenotype of OA-SFs.

\section{MK801's inhibition of OA-SFs inflammatory response depends on C5}

To evaluate the role of C5 in MK801 inhibiting the inflammatory response of OA-SFs. As shown in Figure 4A\&B, we successfully knocked down C5 (sh-C5) and overexpressed C5 (OE-C5) in OA-SFs. MK801 significantly inhibited MAC protein expression levels compared with the control group. However, in the case of C5 knockdown, the inhibitory effect of MK801 on MAC protein expression level disappeared. Compared with the control group, MK801 can significantly inhibit the protein expression level of MAC. However, when C5 is knocked down, the inhibitory effect of MK801 on MAC protein expression level disappears. The MK801's inhibition on MAC protein expression level was not affected when C5 was overexpressed (Figure 4C\&D). Consistent with this MK801 could significantly inhibit the release of TNF- $\alpha$ and IL-1 $\beta$ in comparison to control group, and this inhibition could be abolished by knocking down C5 (Figure 4E\&F). Taken together, these data suggested that MK801's inhibition of OA-SFs inflammatory response depends on C5. 


\section{Discussion}

253

Several reports have shown that MK-801inhibited the activition of NF- $\kappa B$ signaling pathway in the nerve cells $[27,31]$, while there is little published data on the MK-801 effect on NF-KB signaling pathway in OA. Our results showed that MK-801 inhibited the activition of NF- $\mathrm{BB}$ signaling pathway by preventing $\mathrm{p} 65 / \mathrm{p} 50$ from entering the nucleus in OA-SFs. This might also be the reason why MK-801 can inhibit OA-SFs from releasing TNF- $\alpha$ and IL- $1 \beta$.

Honda $\mathrm{K}$ and Fujisawa $\mathrm{T}$, etal. reported that activated synovial macrophages, synovial fibroblasts or chondrocytes themselves induced the expression of MMPs which directly facilitated persistent joint inflammation and joint cartilage destruction in OA[10, 11]. Our data showed MK-801 inhibited MMP-13 expression in OA-SFs, implying the potential of MK801 of inhibiting OA. As mentioned in the literature review, complement activation appears critical in OA pathogenesis resulting the deposition of cell membrane attack complex (MAC) on the surface of chondrocytes[32]. One of our interesting findings is that MK801 can inhibit the formation of MAC on the OA-SFs cell membrane, which further supports the potential of MK801 to inhibit OA.

Previous studies have shown that the formation of MAC is closely associated with $\mathrm{C} 5$, and the formation of MAC requires the participation of the spliceosome of C5[33, 34], so we speculate that MK801's inhibition of the formation of MAC is related to C5. Our data proves this hypothesis: the inhibition of MAC expression by MKK801 in OA-SFs is abolished by knockdown C5. In addition, it is somewhat surprising that our results demonstrate that the inhibition of MK801 on OA-SFs release of OA-related inflammatory factors also depended on C5. This may be due to the involvement of complement system activation in the immune activation of OA and the release of related inflammatory factors[24, 35, 36].

Prior studies that have noted the importance of pro-inflammatory cytokines, complement system and ECM degradation factors in the pathophysiology of OA[10, 11, 21, 22, 37]. These factors are highly expressed in OA-assosiated tissues, the inhibition of these factors can be regarded as a sign of OA inhibition. And our results 
have shown that the antagonist NMDA receptor: MK-801 can signiciantly inhihibited the expression of these factors, implying the possibilty of MK-801 inhibiting OA. Taken together, these findings suggest two roles of MK801 for OA-SFs: first, MK801 inhibited the complement system activation and the release of inflammation factors through C5; second, MK801 inhibited the activation of NF-אB signaling pathway in OA-SFs. These results indicate that MK801 can be used as a potential therapeutic drug for OA, and C5 is also a good therapeutic target in the formation of OA.

\section{Conclusions}

In this stduy, we illustrated two points: first, MK801 inhibited the generation of MAC and the release of inflammation factors in OA-SFs through C5; second: MK801 inhibited the activation of $\mathrm{NF}-\kappa \mathrm{B}$ signaling pathway in OA-SFs. These results suggesting MK801 can be used as a potential therapeutic drug for OA, and C5 is also a good therapeutic target in the formation of OA.

Abbreviations: extracellular cartilage matrix (ECM), interleukin-1 $\beta$ (IL-1 $\beta$ ), tumor necrosis factor- $\alpha$ (TNF- $\alpha$ ), matrix metalloproteinases (MMP), complement C5 (C5), osteoarthritis synovial fibroblasts (OA-SFs), osteoarthritis (OA), membrane attack complex (MAC), Nuclear factor- $\kappa \mathrm{B}(\mathrm{NF}-\kappa \mathrm{B})$, fibroblast-like synovial cells (FLS) and N-Methyl-D-aspartate (NMDA)

\section{Declarations}

\section{Ethics approval and consent to participate}

All mice were housed in a pathogen-free environment at the Guizhou Medical University. All experimental protocols were approved by the Institutional Committee for Animal Care and Use at Guizhou Medical University. All animal work was performed in accordance with the approved protocol.

\section{Consent for publication}

Written informed consent for publication was obtained from all participants. 
312 All data generated or analyzed during this study are included in this article.

313 Competing interests

314 The authors declare no conflict of interest.

\section{Funding}

316 This work was financially supported by Guizhou Province Science and technology

317 plan project (talents of qiankehe platform [2018] 5779-50) and the Science and

318 technology plan of Guiyang science and Technology Bureau, Zhuke contract [2017]

319 No. 30-35.

320 Authors' contributions

321 Z. H., and Y. F.performed the experiments. L. W. provided helpful comments. W.L.

322 conceived the work. W.L. wrote the manuscript. And all authors have read and

323 approved the manuscript

\section{Acknowledgements}

325 The authors would like to acknowledge the Guizhou Province Science and technology 326 plan project (talents of qiankehe platform [2018] 5779-50) and the Science and 327 technology plan of Guiyang science and Technology Bureau, Zhuke contract [2017] 328 No. 30-35. 


\section{References}

1. Felson DT. Developments in the clinical understanding of osteoarthritis. Arthritis Research \& Therapy 2009,11.

2. Johnston SA. Osteoarthritis. Joint anatomy, physiology, and pathobiology. Vet Clin North Am Small Anim Pract 1997,27:699-723.

3. Dingle JT. Catabolin--a cartilage catabolic factor from synovium. Clin Orthop Relat Res 1981:219-231.

4. Ushiyama T, Chano T, Inoue K, Matsusue Y. Cytokine production in the infrapatellar fat pad: another source of cytokines in knee synovial fluids. Ann Rheum Dis 2003,62:108-112.

5. Hayashi K, Frank JD, Dubinsky C, Zhengling H, Markel MD, Manley PA, et al. Histologic changes in ruptured canine cranial cruciate ligament. Vet Surg 2003,32:269-277.

6. Rogers J, Shepstone L, Dieppe P. Is osteoarthritis a systemic disorder of bone? Arthritis Rheum 2004,50:452-457.

7. Brandt KD, Myers SL, Burr D, Albrecht M. Osteoarthritic changes in canine articular cartilage, subchondral bone, and synovium fifty-four months after transection of the anterior cruciate ligament. Arthritis Rheum 1991,34:1560-1570.

8. Pelletier JP, Martel-Pelletier J, Abramson SB. Osteoarthritis, an inflammatory disease: potential implication for the selection of new therapeutic targets. Arthritis Rheum 2001,44:1237-1247.

9. Feldmann M. Development of anti-TNF therapy for rheumatoid arthritis. Nat Rev Immunol 2002,2:364-371.

10. Honda K, Ohno S, Tanimoto K, Ijuin C, Tanaka N, Doi T, et al. The effects of high magnitude cyclic tensile load on cartilage matrix metabolism in cultured chondrocytes. Eur J Cell Biol 2000,79:601-609.

11. Fujisawa T, Hattori T, Takahashi K, Kuboki T, Yamashita A, Takigawa M. Cyclic mechanical stress induces extracellular matrix degradation in cultured chondrocytes via gene expression of matrix metalloproteinases and interleukin-1. J Biochem 1999,125:966-975.

12. Malemud CJ. Biologic basis of osteoarthritis: state of the evidence. Curr Opin Rheumatol 2015,27:289-294.

13. Goldring SR, Goldring MB. Changes in the osteochondral unit during osteoarthritis: structure, function and cartilage-bone crosstalk. Nat Rev Rheumatol 2016,12:632-644.

14. Kapoor M, Martel-Pelletier J, Lajeunesse D, Pelletier JP, Fahmi H. Role of proinflammatory cytokines in the pathophysiology of osteoarthritis. Nature Reviews Rheumatology 2011,7:33-42.

15. Fernandes JC, Martel-Pelletier J, Pelletier JP. The role of cytokines in osteoarthritis pathophysiology. Biorheology 2002,39:237-246.

16. Roman-Blas JA, Stokes DG, Jimenez SA. Modulation of TGF-beta signaling by proinflammatory cytokines in articular chondrocytes. Osteoarthritis and Cartilage 2007,15:1367-1377.

17. Smith MD, Triantafillou S, Parker A, Youssef PP, Coleman M. Synovial membrane inflammation and cytokine production in patients with early osteoarthritis. J Rheumatol 1997,24:365-371.

18. Riyazi N, Slagboom E, de Craen AJ, Meulenbelt I, Houwing-Duistermaat JJ, Kroon HM, et al. Association of the risk of osteoarthritis with high innate production of interleukin-1beta and low innate production of interleukin-10 ex vivo, upon lipopolysaccharide stimulation. Arthritis Rheum 2005,52:1443-1450. 
19. Pelletier JP, Roughley PJ, DiBattista JA, McCollum R, Martel-Pelletier J. Are cytokines involved in osteoarthritic pathophysiology? Semin Arthritis Rheum 1991,20:12-25.

20. Liu-Bryan R, Terkeltaub R. Emerging regulators of the inflammatory process in osteoarthritis. Nat Rev Rheumatol 2015,11:35-44.

21. Wang Q, Rozelle AL, Lepus CM, Scanzello CR, Song JJ, Larsen DM, et al. Identification of a central role for complement in osteoarthritis. Nature Medicine 2011,17:1674-U1196.

22. Joos H, Leucht F, Riegger J, Hogrefe C, Fiedler J, Durselen L, et al. Differential Interactive Effects of Cartilage Traumatization and Blood Exposure In Vitro and In Vivo. American Journal of Sports Medicine 2015,43:2822-2832.

23. Malemud CJ. Biologic basis of osteoarthritis: state of the evidence. Current Opinion in Rheumatology 2015,27:289-294.

24. Blom AM. The role of complement inhibitors beyond controlling inflammation. Journal of Internal Medicine 2017,282:116-128.

25. Cai Y, Ford CP. Dopamine Cells Differentially Regulate Striatal Cholinergic Transmission across Regions through Corelease of Dopamine and Glutamate. Cell Reports 2018,25:3148-+.

26. O'Donnell JC, Jackson JG, Robinson MB. Transient Oxygen/Glucose Deprivation Causes a Delayed Loss of Mitochondria and Increases Spontaneous Calcium Signaling in Astrocytic Processes. Journal of Neuroscience 2016,36:7109-7127.

27. Kawamoto EM, Lima LS, Munhoz CD, Yshii LM, Kinoshita PF, Amaral FG, et al. Influence of $\mathrm{N}$-methyl-D-aspartate receptors on ouabain activation of nuclear factor-kappa $\mathrm{B}$ in the rat hippocampus. Journal of Neuroscience Research 2012,90:213-228.

28. Zhao J, Ouyang Q, Hu Z, Huang Q, Wu J, Wang R, et al. A protocol for the culture and isolation of murine synovial fibroblasts. Biomed Rep 2016,5:171-175.

29. Kawamoto EM, Lima LS, Munhoz CD, Yshii LM, Kinoshita PF, Amara FG, et al. Influence of $\mathrm{N}$-methyl-D-aspartate receptors on ouabain activation of nuclear factor-kappaB in the rat hippocampus. J Neurosci Res 2012,90:213-228.

30. Silawal S, Triebel J, Bertsch T, Schulze-Tanzil G. Osteoarthritis and the Complement Cascade. Clin Med Insights Arthritis Musculoskelet Disord 2018,11:1179544117751430.

31. Ye L, Huang YL, Zhao LX, Li YJ, Sun LJ, Zhou Y, et al. IL-1 and TNF- induce neurotoxicity through glutamate production: a potential role for neuronal glutaminase. Journal of Neurochemistry 2013,125:897-908.

32. Silawal S, Triebel J, Bertsch T, Schulze-Tanzil G. Osteoarthritis and the Complement Cascade. Clinical Medicine Insights-Arthritis and Musculoskeletal Disorders 2018,11.

33. Carroll MV, Sim RB. Complement in health and disease. Advanced Drug Delivery Reviews 2011,63:965-975.

34. Woodruff TM, Nandakumar KS, Tedesco F. Inhibiting the C5-C5a receptor axis. Molecular Immunology 2011,48:1631-1642.

35. Onuma H, Masuko-Hongo K, Yuan GH, Sakata M, Nakamura H, Kato T, et al. Expression of the anaphylatoxin receptor $\mathrm{C} 5 \mathrm{aR}(\mathrm{CD} 88)$ by human articular chondrocytes. Rheumatology International 2002,22:52-55.

36. Kim DD, Song WC. Membrane complement regulatory proteins. Clinical Immunology 2006,118:127-136.

37. Wang TT, He CQ. Pro-inflammatory cytokines: The link between obesity and osteoarthritis. Cytokine \& Growth Factor Reviews 2018,44:38-50. 


\section{Figure legend}

Figure 1. MK801 inhibited the expression of OA marker factors in OA-SFs

A: The expression levels of MAC, TNF- $\alpha$, C5 and MMP13 protein were dectected by western blot in OA-SFs treated with vehicle, $20 \mathrm{nM}$ MK801 or $100 \mu \mathrm{M}$ NMDA for 24 hours, indicated antibodies were added during western blot assay, Full-length blots/gels are presented in Supplementary Figure 1; B: Statistical analysis of the MAC, TNF- $\alpha$, C5 and MMP13 protein levels based on western blot assay results; C: The expression levels of TNF- $\alpha$, C5 and MMP13 mRNA were dectected by RT-PCR in OA-SFs treated with vehicle, $20 \mathrm{nM}$ MK801 or $100 \mu \mathrm{M}$ NMDA for 24 hours; D: Statistical analysis of the TNF- $\alpha$, C5 and MMP13 mRNA levels based on RT-PCR assay results; E\&F: TNF- $\alpha$ and IL-1 $\beta$ protein levels in OA-SFs cell culture medium after treating with vehicle, $20 \mathrm{nM}$ MK801 or $100 \mu \mathrm{M}$ NMDA for 24 hours were detected by .

Data are representative of three independent experiments, and were analyzed by unpaired t-test. Error bars denote SD. $* P<0.05 ; * * P<0.01 ; * * * P<0.001$ Figure 2. MK801 inhibited the activation of NF- $\mathrm{KB}$ signaling pathway in OA-SFs A: Cytoplasm and nucleus p65/p50 protein levels in the cytoplasm dectected by western blot after nucleocytoplasmic separation of OA-SFs (treated with vehicle, 20 nM MK801 or $100 \mu \mathrm{M}$ NMDA for 24 hours), indicated antibodies were added during western blot assay, Full-length blots/gels are presented in Supplementary Figure 2; B: Statistical analysis of the p65/p50 protein levels based on western blot assay results. Data are representative of three independent experiments, and were analyzed by unpaired t-test. Error bars denote SD. $* P<0.05$; $* * P<0.01$; $* * * P<0.001$

\section{Figure 3. MK801 inhibited MAC expression on OA-SFs cell membrane}

A: MAC protein expression level on the OA-SFs cell membrane was detected by immunofluorescence assay after treating with vehicle or $20 \mathrm{nM}$ MK801 for 24 hours, indicated antibodies were added during immunofluorescence assay, scale bars: $50 \mu \mathrm{m}$; B: Statistical analysis of the OA-SFs cell membrane MAC protein levels based on immunofluorescence assay results; C: Simplified diagram of the relationship between 
complement C5 and MAC.

Data are representative of three independent experiments, and were analyzed by unpaired t-test. Error bars denote SD. $* P<0.05 ; * * P<0.01 ; * * * P<0.001$

Figure 4. MK801's inhibition of OA-SFs inflammatory response depends on C5

A: The expression level of C5 in OA-SFs was dectected by western blot after indicated plasmids were transfected in OA-SFs, indicated antibodies were added during western blot assay, Full-length blots/gels are presented in Supplementary Figure 3; B: Statistical analysis of the C5 protein level based on western blot assay results after indicated plasmids were transfected in OA-SFs, Full-length blots/gels are presented in Supplementary Figure 4; C: MK801's impact on OA-SFs MAC protein expression level was dectected by western blot after indicated plasmids were transfected in OA-SFs, indicated antibodies were added during western blot assay; D: Statistical analysis of MAC protein expression level based on western blot assay results; E\&F: MK801's impact on TNF- $\alpha$ and IL-1 $\beta$ protein levels in OA-SFs cell culture medium were detected by ELISA assay after indicated plasmids were transfected in OA-SFs.

Data are representative of three independent experiments, and were analyzed by unpaired t-test. Error bars denote SD. $* P<0.05 ; * * P<0.01 ; * * * P<0.001$ 
479 Figure 1. MK801 inhibited the expression of OA marker factors in OA-SFs

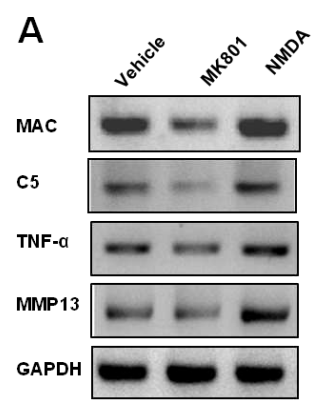

D

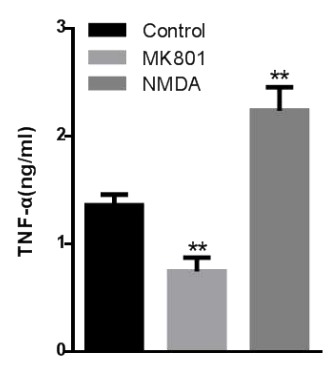

B

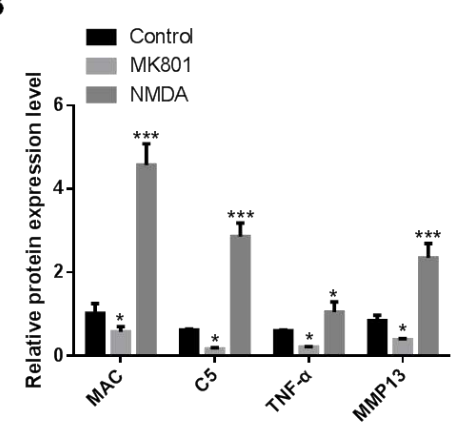

E

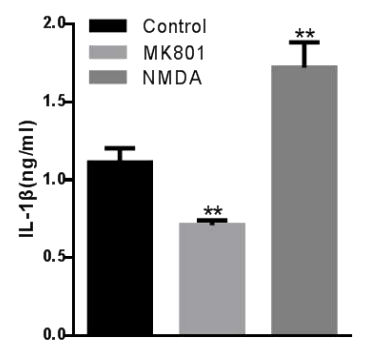

C

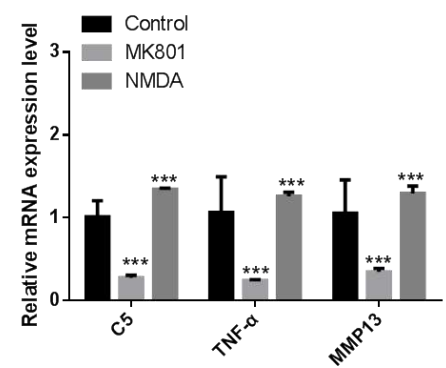

480

481

482 
Figure 2. MK801 inhibited the activation of NF-kB signaling pathway in OA-SFs

A

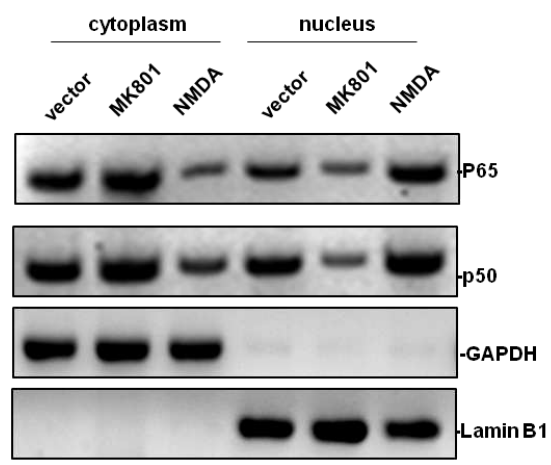

B

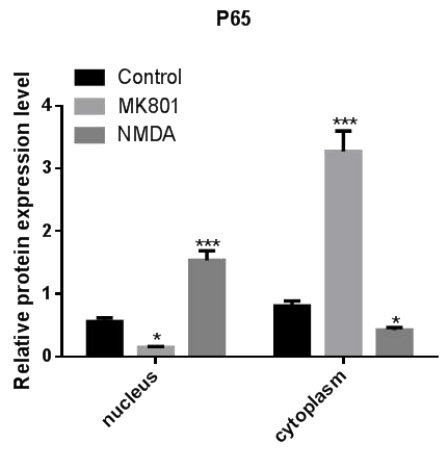

P50

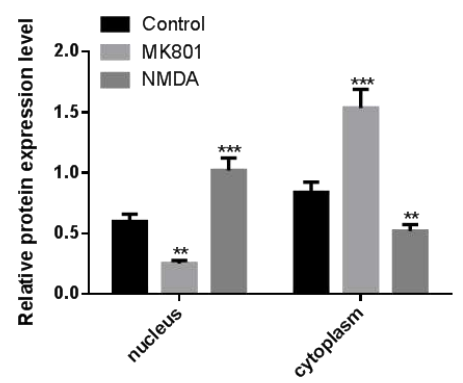

485

486

487

488

489

490 
491 Figure 3. MK801 inhibited MAC expression on OA-SFs cell membrane

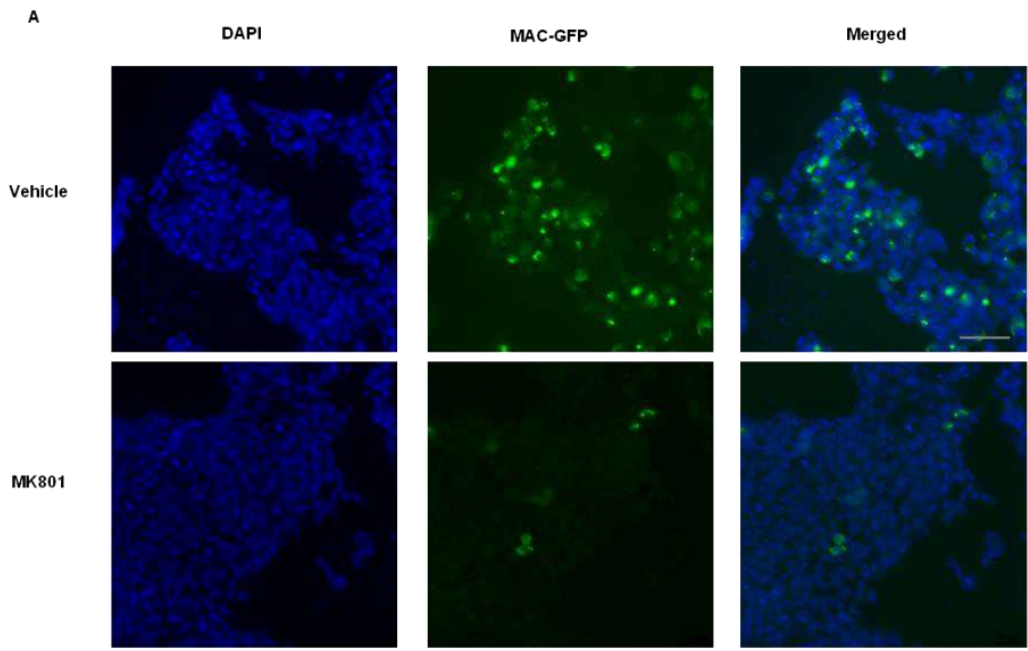

B

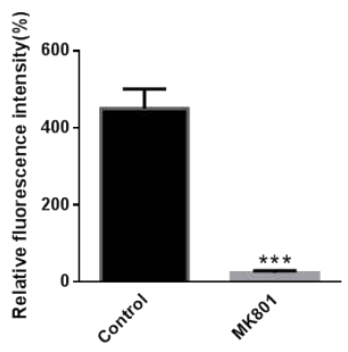

c

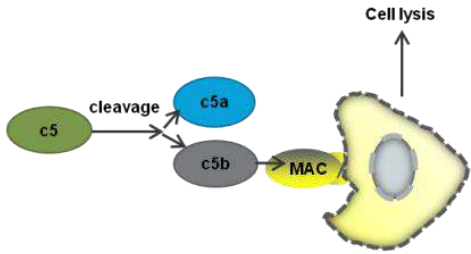

492

493 
Figure 4. MK801's inhibition of OA-SFs inflammatory response depends on C5
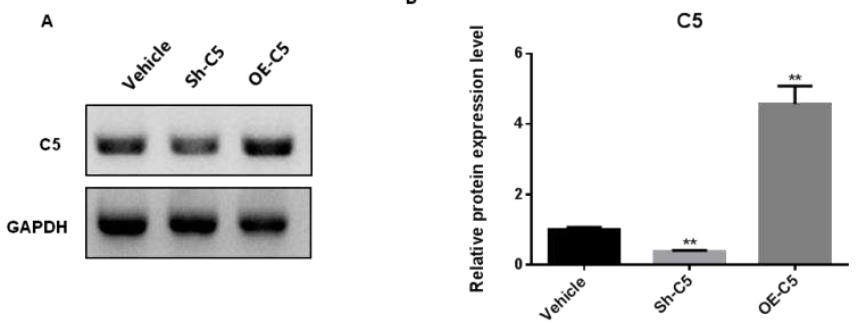

c
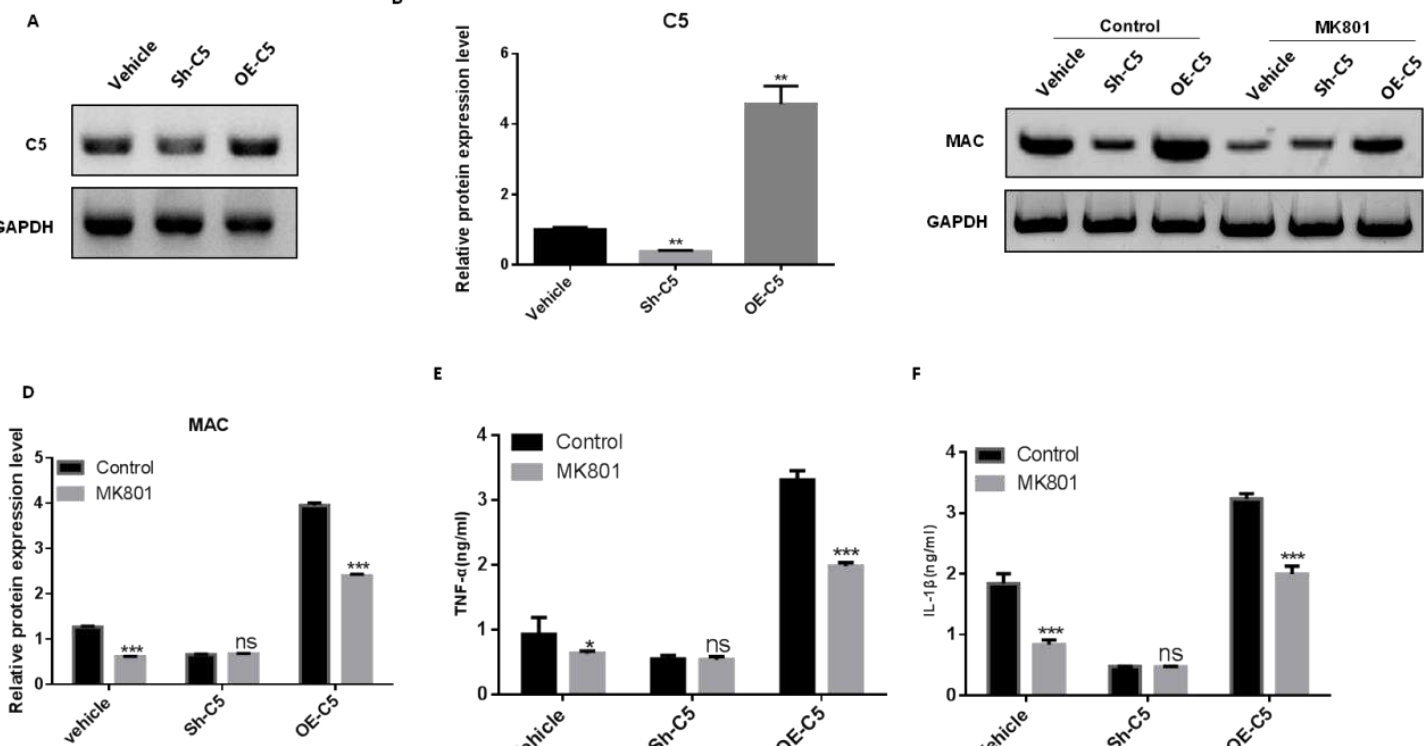

E
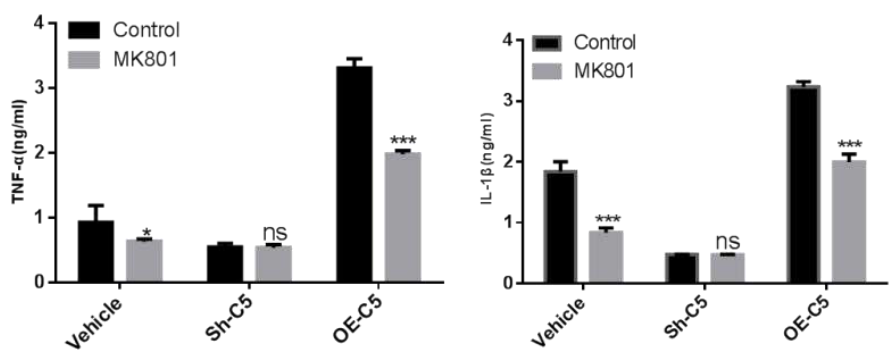

495 


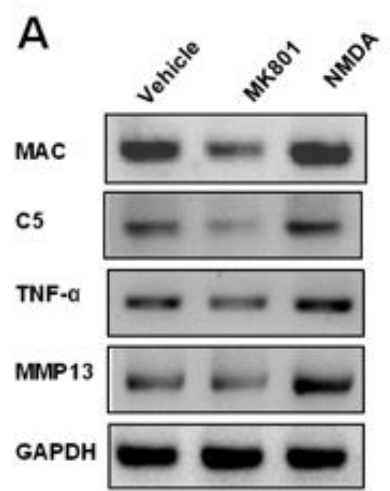

D

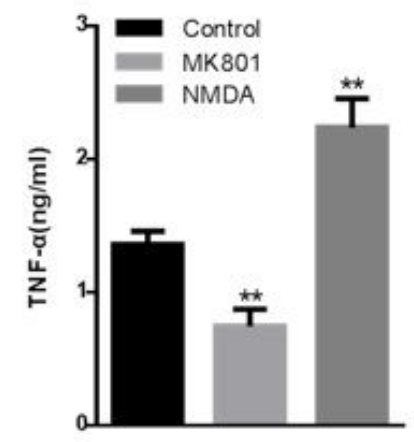

B

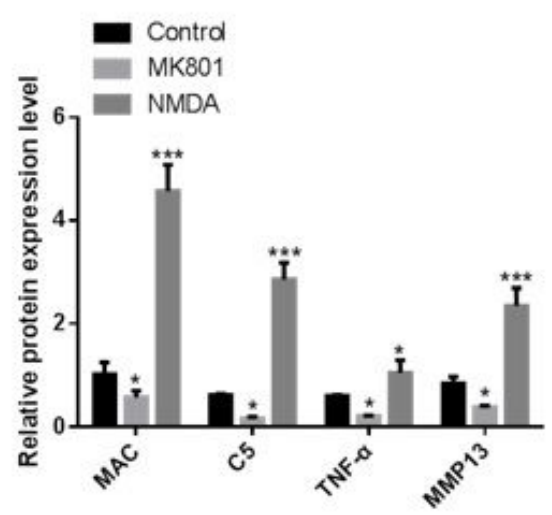

E

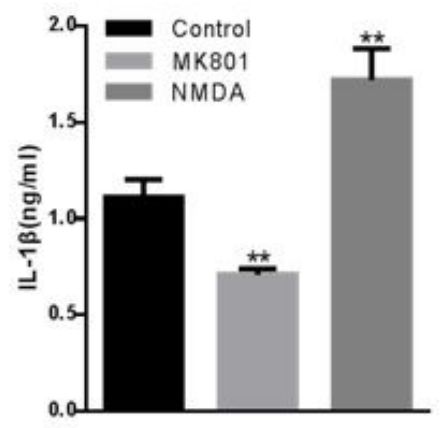

C

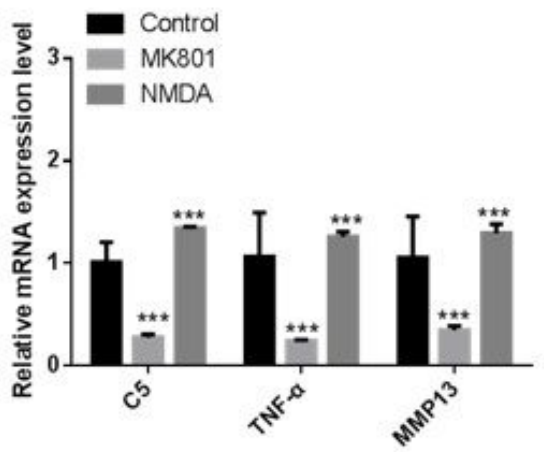

\section{Figure 1}

MK801 inhibited the expression of OA marker factors in OA-SFs A: The expression levels of MAC, TNF-a, C5 and MMP13 protein were dectected by western blot in OA-SFs treated with vehicle, $20 \mathrm{nM}$ MK801 or $100 \mu \mathrm{M}$ NMDA for 24 hours, indicated antibodies were added during western blot assay, Full-length blots/gels are presented in Supplementary Figure 1; B: Statistical analysis of the MAC, TNF-a, C5 and MMP13 protein levels based on western blot assay results; $C$ : The expression levels of TNF-a, $C 5$ and MMP13 mRNA were dectected by RT-PCR in OA-SFs treated with vehicle, $20 \mathrm{nM}$ MK801 or $100 \mu \mathrm{M}$ NMDA for 24 hours; D: Statistical analysis of the TNF-a, C5 and MMP13 mRNA levels based on RT-PCR assay results; E\&F: TNF- $\alpha$ and IL-1 $\beta$ protein levels in OA-SFs cell culture medium after treating with vehicle, 20 nM MK801 or $100 \mu \mathrm{M}$ NMDA for 24 hours were detected by . Data are representative of three independent experiments, and were analyzed by unpaired t-test. Error bars denote SD. ${ }^{\star} P<0.05$; ${ }^{\star \star P}<0.01$; $* \star \star P<$ 0.001 
A

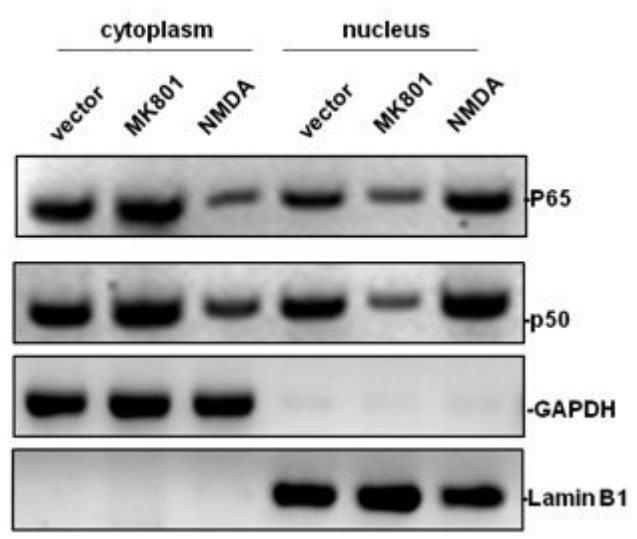

B

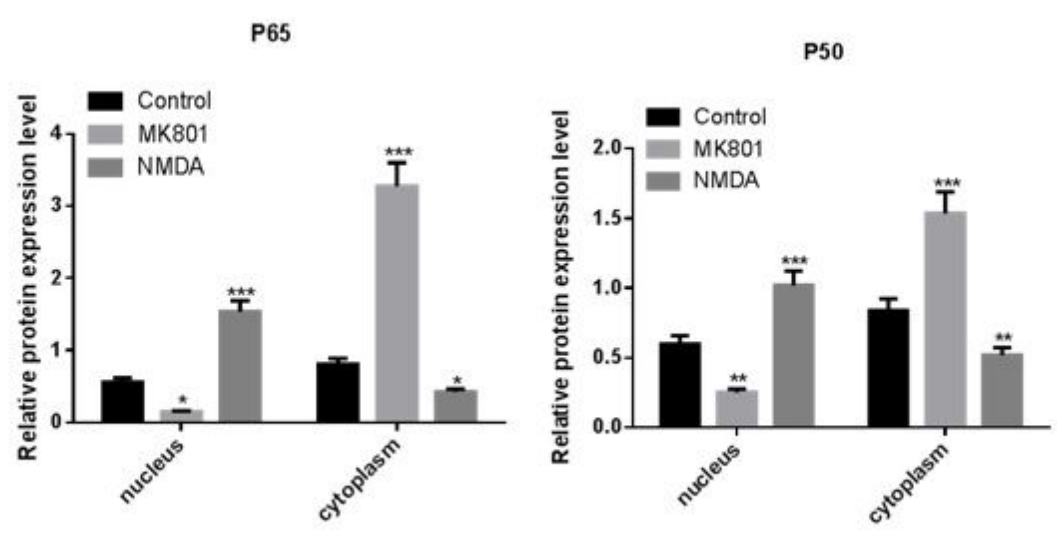

\section{Figure 2}

MK801 inhibited the activation of NF-KB signaling pathway in OA-SFs A: Cytoplasm and nucleus p65/p50 protein levels in the cytoplasm dectected by western blot after nucleocytoplasmic separation of OA-SFs (treated with vehicle, $20 \mathrm{nM}$ MK801 or $100 \mu \mathrm{M}$ NMDA for 24 hours), indicated antibodies were added during western blot assay, Full-length blots/gels are presented in Supplementary Figure 2; B: Statistical analysis of the p65/p50 protein levels based on western blot assay results. Data are representative of three independent experiments, and were analyzed by unpaired t-test. Error bars denote $S D$. ${ }^{\star} P<0.05$; $* \star P$ $<0.01 ; * \star * P<0.001$

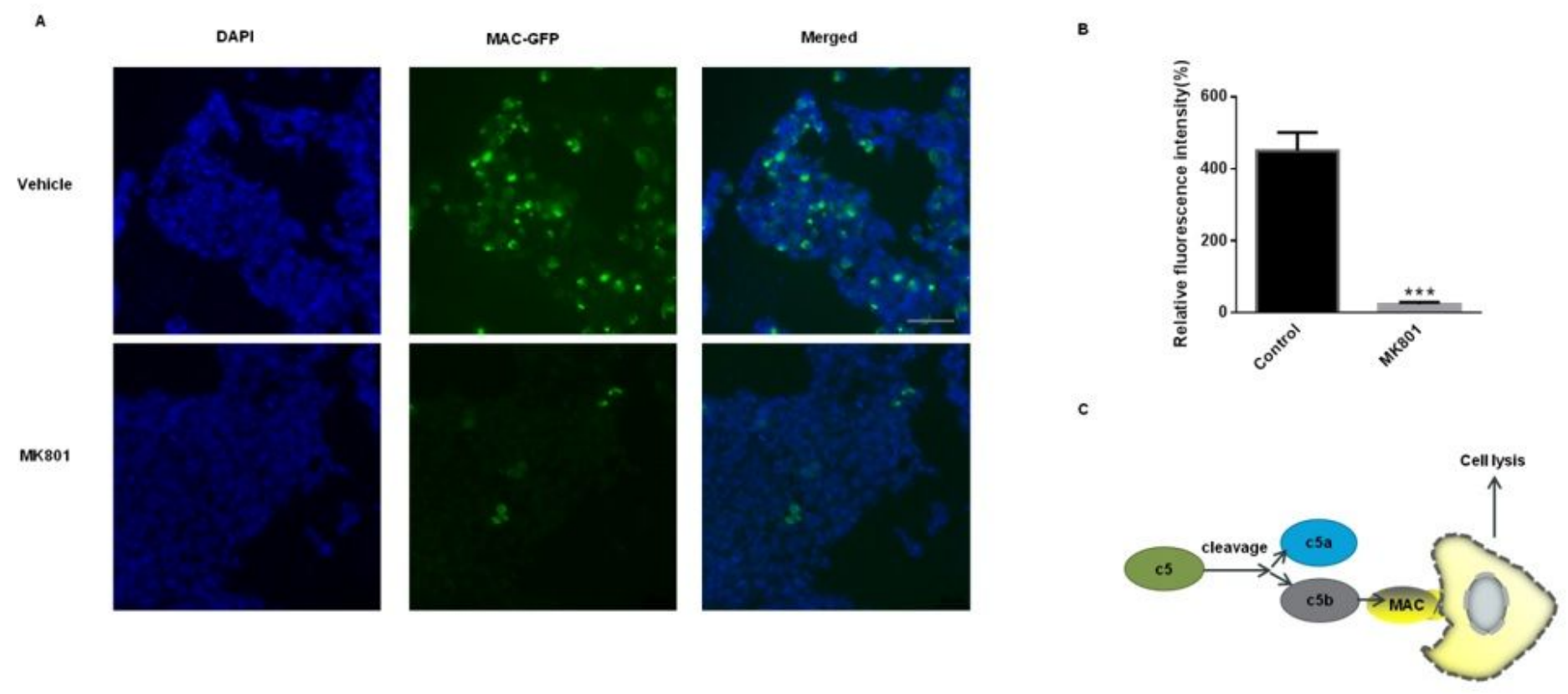

Figure 3

MK801 inhibited MAC expression on OA-SFs cell membrane A: MAC protein expression level on the OASFs cell membrane was detected by immunofluorescence assay after treating with vehicle or $20 \mathrm{nM}$ MK801 for 24 hours, indicated antibodies were added during immunofluorescence assay, scale bars: 50 
$\mu \mathrm{m} ; \mathrm{B}$ : Statistical analysis of the OA-SFs cell membrane MAC protein levels based on immunofluorescence assay results; C: Simplified diagram of the relationship between complement C5 and MAC. Data are representative of three independent experiments, and were analyzed by unpaired ttest. Error bars denote SD. ${ }^{*} \mathrm{P}<0.05 ; * \star \mathrm{P}<0.01 ; * \star * \mathrm{P}<0.001$
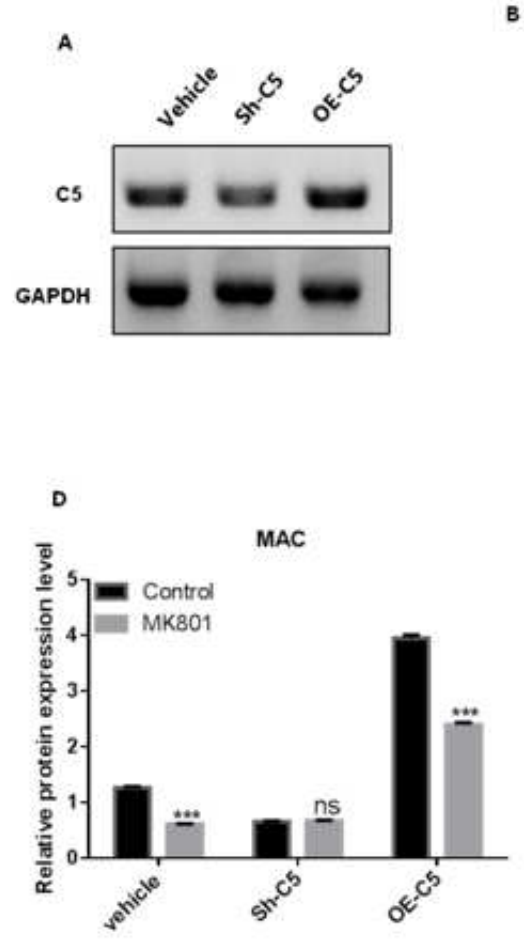

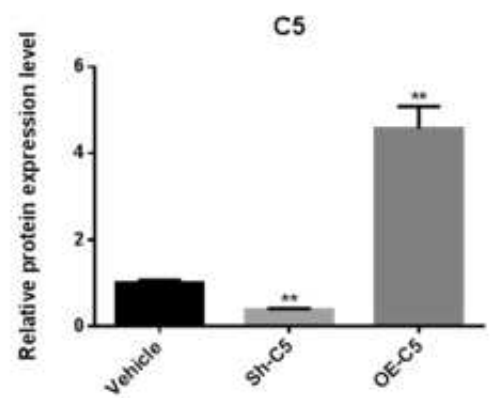

E

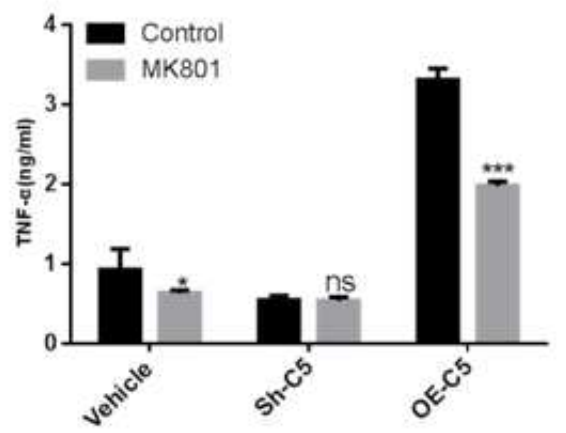

c
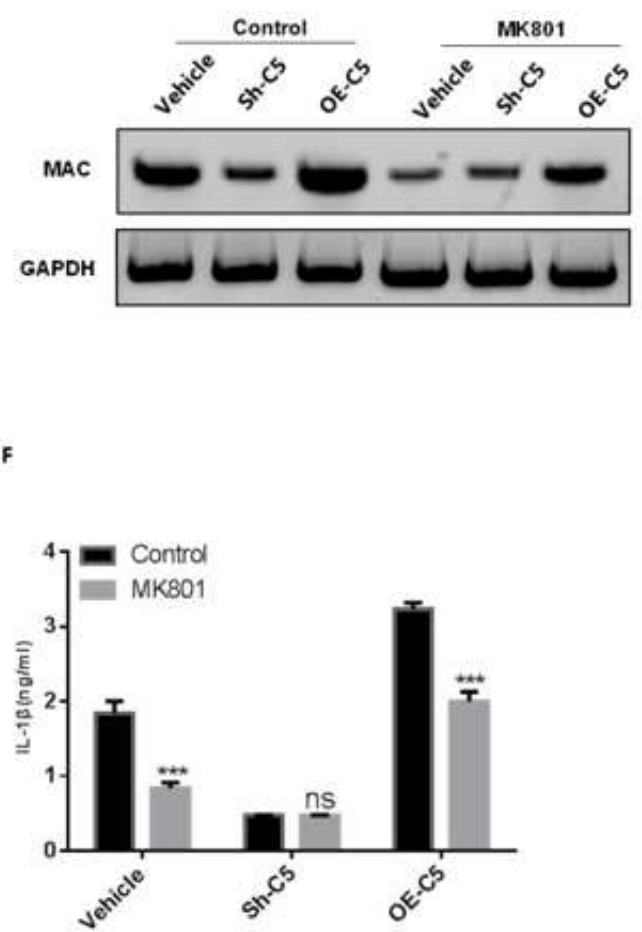

\section{Figure 4}

MK801's inhibition of OA-SFs inflammatory response depends on C5 A: The expression level of C5 in OASFs was dectected by western blot after indicated plasmids were transfected in OA-SFs, indicated antibodies were added during western blot assay, Full-length blots/gels are presented in Supplementary Figure 3; B: Statistical analysis of the C5 protein level based on western blot assay results after indicated plasmids were transfected in OA-SFs, Full-length blots/gels are presented in Supplementary Figure 4; C: MK801's impact on OA-SFs MAC protein expression level was dectected by western blot after indicated plasmids were transfected in OA-SFs, indicated antibodies were added during western blot assay; D: Statistical analysis of MAC protein expression level based on western blot assay results; E\&F: MK801's impact on TNF- $\alpha$ and IL-1 $\beta$ protein levels in OA-SFs cell culture medium were detected by ELISA assay after indicated plasmids were transfected in OA-SFs. Data are representative of three independent experiments, and were analyzed by unpaired t-test. Error bars denote $S D$. ${ }^{\star} P<0.05$; ${ }^{\star \star P}<0.01$; ${ }^{\star \star \star P} P<$ 0.001

\section{Supplementary Files}

This is a list of supplementary files associated with this preprint. Click to download. 
- ARRIVEchecklist.pdf

- SupplementaryFigure1.tif

- SupplementaryFigure2.tif

- SupplementaryFigure3.tif

- SupplementaryFigure4.tif 